Physics

Physics Research Publications

\title{
Spin-glass ordering in the layered III-VI diluted magnetic semiconductor $\mathrm{Ga}(1-\mathrm{x}) \mathrm{MnxS}$
}
T. M. Pekarek
E. M. Watson
P. M. Shand
I. Miotkowski
A. K. Ramdas

This paper is posted at Purdue e-Pubs.

http://docs.lib.purdue.edu/physics_articles/1309 


\title{
Spin-glass ordering in the layered III-VI diluted magnetic semiconductor $\mathrm{Ga}_{1-x} \mathrm{Mn}_{x} \mathrm{~S}$
}

\author{
T. M. Pekarek, ${ }^{1, a)}$ E. M. Watson, ${ }^{1}$ P. M. Shand ${ }^{2}$ I. Miotkowski, ${ }^{3}$ and A. K. Ramdas ${ }^{3}$ \\ ${ }^{1}$ Department of Physics, University of North Florida, Jacksonville, Florida 32224, USA \\ ${ }^{2}$ Department of Physics, University of Northern Iowa, Cedar Falls, Iowa 50614, USA \\ ${ }^{3}$ Department of Physics, Purdue University, West Lafayette, Indiana 47907, USA
}

(Presented 21 January 2010; received 31 October 2009; accepted 26 January 2010; published online 5 May 2010)

\begin{abstract}
Magnetization and ac susceptibility measurements on single crystalline $\mathrm{Ga}_{1-x} \mathrm{Mn}_{x} \mathrm{~S}$ ( $x=0.09$ ) have been measured near the spin-glass transition. No other III-VI diluted magnetic semiconductor (DMS) is currently known to exhibit a spin-glass transition for comparison with $\mathrm{Ga}_{1-x} \mathrm{Mn}_{x} \mathrm{~S}$. Both the magnetization and ac susceptibility measurements were analyzed according to the appropriate universal scaling function for spin-glass transitions. The nonlinear magnetization scaled with the critical exponent values $\gamma=4.0 \pm 1.0$ and $\beta=0.8 \pm 0.2$ for a spin-glass transition temperature $T_{\mathrm{c}}$ $=11.2 \pm 0.2 \mathrm{~K}$. The analysis of the ac susceptibility's out-of-phase component $\chi^{\prime \prime}(\omega, T)$ yielded the parameter values $T_{\mathrm{c}}=10.8 \pm 0.3 \mathrm{~K}, z \nu=10 \pm 1$, and $\beta=0.6 \pm 0.3$. The values of the critical exponents are consistent with those obtained in many DMS and non-DMS insulating spin glasses with different lattice structures and exchange interactions. These results indicate that III-V DMS materials such as $\mathrm{Ga}_{1-x} \mathrm{Mn}_{x} \mathrm{~S}$ belong in the same three-dimensional short-range Heisenberg universality class as other DMS and non-DMS insulating spin glasses. (C) 2010 American Institute of Physics. [doi:10.1063/1.3366616]
\end{abstract}

$\mathrm{Ga}_{1-x} \mathrm{Mn}_{x} \mathrm{~S}$ is in the class of layered III-VI diluted magnetic semiconductors (DMSs). The two-dimensional crystal structure of $\mathrm{Ga}_{1-x} \mathrm{Mn}_{x} \mathrm{~S}$ (inset of Fig. 1) is confined by van der Waals bonds above and below the 4 atom thick layer. Superexchange between two $\mathrm{Mn}$ ions is mediated by a $\mathrm{S}$ ion in the top and bottom capping layers (Mn-S-Mn) similar to the II-VI DMS. However, more complex exchange channels such as $\mathrm{Mn}-\mathrm{S}-\mathrm{Ga}-\mathrm{Mn}$ or direct $\mathrm{Mn}-\mathrm{Mn}$ bonds are available in the III-VI DMS systems.

The III-VI semiconductors, such as $\mathrm{GaSe},{ }^{1-4} \mathrm{InSe},{ }^{4,5}$ $\mathrm{GaTe},{ }^{6}$ and $\mathrm{GaS},{ }^{7-9}$ have received considerable interest because of their remarkable nonlinear optical properties. Work on $\mathrm{GaS}$ includes growth of GaS films and multilayers on a GaAs substrate, ${ }^{8,10}$ thermal conductivity, ${ }_{11}^{11}$ and fabrication of Ohmic contacts to $n$-GaAs using GaS. ${ }^{9,12}$ Suhre et al. ${ }^{3}$ have shown that adding In to GaSe strengthens the material enough to polish optical faces along arbitrary crystalline directions while enhancing the optical characteristics.

Recently Mn and Fe have been incorporated to form the III-VI DMS $\mathrm{Ga}_{1-x} \mathrm{Mn}_{x} \mathrm{~S},{ }^{13-15} \mathrm{In}_{1-x} \mathrm{Mn}_{x} \mathrm{~S},{ }^{16} \mathrm{In}_{1-x} \mathrm{Mn}_{x} \mathrm{Se},{ }^{17,18}$ $\mathrm{Ga}_{1-x} \mathrm{Mn}_{x} \mathrm{Se},{ }^{1}$ and $\mathrm{Ga}_{1-x} \mathrm{Fe}_{x} \mathrm{Se} .{ }^{19}$ The magnetization behavior of isolated $\mathrm{Mn}$ ions and $\mathrm{Mn}$ pairs in $\mathrm{Ga}_{1-x} \mathrm{Mn}_{x} \mathrm{~S}$ from 50 to $400 \mathrm{~K}$ in fields up to 70000 Oe has been investigated previously ${ }^{13-15}$ and is well understood and is similar to the II-VI DMS. ${ }^{14,16}$ In this region, the magnetization arises from substitutional Mn ions at the Ga lattice site with an increasing importance of the antiferromagnetic interaction of $\mathrm{Mn}$ nearest neighbors below $50 \mathrm{~K}$. While spin-glass transitions are known to exist in a number of systems ${ }^{20,21}$ including

${ }^{\text {a)} E l e c t r o n i c ~ m a i l: ~ t p e k a r e k @ u n f . e d u . ~}$
II-VI DMS such as $\mathrm{Zn}_{1-x} \mathrm{Mn}_{x} \mathrm{Te},{ }^{22}$ no III-VI DMS has been reported to exhibit a spin-glass transition except for $\mathrm{Ga}_{1-x} \mathrm{Mn}_{x} \mathrm{~S}^{23}$

In this work, we present magnetization and ac susceptibility measurements investigating the spin-glass transition in the layered III-VI DMS $\mathrm{Ga}_{1-x} \mathrm{Mn}_{x} \mathrm{~S}$. Since no other III-VI DMS is currently known to exhibit a spin-glass transition, we continue to study this spin-glass system to help determine where it fits into the rich collection of other known spinglass materials.

Magnetization measurements were performed using a Quantum Design superconducting quantum interference device magnetometer on a $0.0160 \mathrm{~g}$ single-crystalline samples of $\mathrm{Ga}_{0.91} \mathrm{Mn}_{0.09} \mathrm{~S}$ grown by the vertical Bridgman method. Measurements for the nonlinear scaling analysis were made

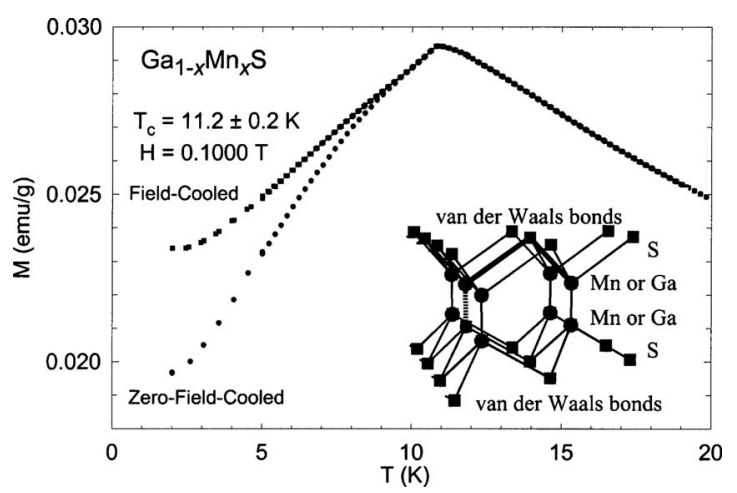

FIG. 1. Zero-field-cooled and field-cooled magnetization vs temperature for $\mathrm{Ga}_{1-x} \mathrm{Mn}_{x} \mathrm{~S}$ are shown by the solid circles and squares, respectively. The diamagnetic contribution has been subtracted. The cusp in the magnetization near $T=11.2 \mathrm{~K}$ for the zero-field-cooled case is characteristic of a transition to the spin-glass phase. 


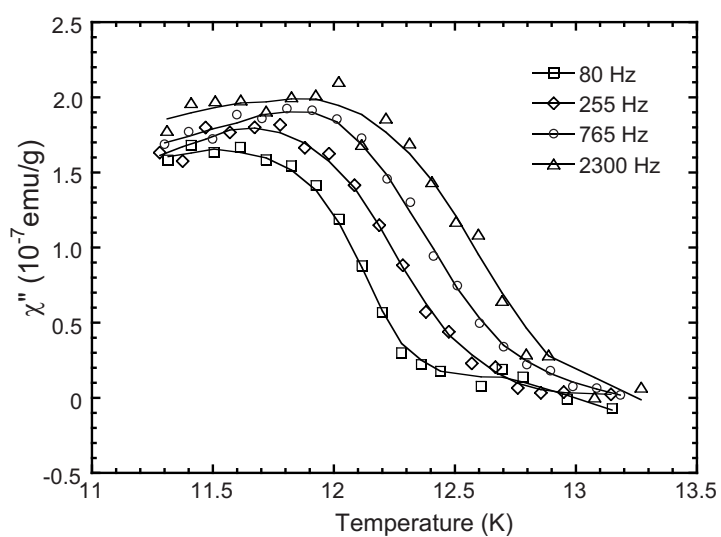

FIG. 2. Imaginary part of the ac susceptibility of $\mathrm{Ga}_{1-x} \mathrm{Mn}_{x} \mathrm{~S}$ as a function of temperature at various frequencies. The solid lines are smoothing curves that enable a more precise determination of the position of the peak for a given frequency. Only data at temperatures greater than that at the peak are used for scaling analysis.

in fields between 0 and 10000 Oe after quenching the superconducting magnet to remove any remanent field. Temperature stability was achieved to within $0.01 \mathrm{~K}$.

The ac magnetic susceptibility measurements on a $0.4379 \mathrm{~g}$ sample were carried out with a LakeShore Model 7225 susceptometer, using an ac field strength of 10 Oe. The superconducting magnet was disconnected so that the only dc field applied to the sample was that of the Earth $(<0.5 \mathrm{Oe})$. The frequencies used in these measurements were $80,255,765$, and $2300 \mathrm{~Hz}$. In the temperature range of our measurements $(10-15 \mathrm{~K})$, the accuracy of the temperature calibration was $\pm 0.1 \mathrm{~K}$. The stability of the temperature was $\pm 0.05 \mathrm{~K}$ during each measurement of the in-phase $\left(\chi^{\prime}\right)$ and out-of-phase $\left(\chi^{\prime \prime}\right)$ components.

Magnetization versus temperature data for $\mathrm{Ga}_{1-x} \mathrm{Mn}_{x} \mathrm{~S}$ $(x=0.09)$ in 1000 Oe is shown in Fig. 1. The prominent cusp as well as splitting between the field-cooled and zero-fieldcooled magnetization are clearly seen. Previous work has investigated the behavior of this peak with increasing field. ${ }^{23}$ It was found that below $1000 \mathrm{Oe}$, the temperature of the cusp at $10.9 \mathrm{~K}$ remained constant. As expected, this temperature is slightly lower than the true spin-glass transition temperature $T_{\mathrm{c}}=11.2 \pm 0.2 \mathrm{~K}$ for this sample because the divergent negative nonlinear magnetization suppresses the high temperature side of the cusp and reduces the temperature at which the cusp appears in the magnetization even in the zero-field limit.

The dynamics of this transition can be studied by the measurement and analysis of the ac susceptibility's out-ofphase component $\chi^{\prime \prime}(\omega, T) .^{24}$ Figure 2 shows $\chi^{\prime \prime}(\omega, T)$ for four different frequencies for the $\mathrm{Ga}_{1-x} \mathrm{Mn}_{x} \mathrm{~S}$ sample. As can be seen in the figure, the temperature at which the peak in $\chi^{\prime \prime}(\omega, T)$ occurs shifts to higher temperatures with increasing applied frequency. This is a well-known feature of systems undergoing relaxation processes, including spin glasses. Phenomenologically, the average size and relaxation time of the clusters of spins constituting a spin glass decrease as the temperature increases. Clusters with relaxation times longer than the period of the driving ac field are effectively blocked and do not contribute to the response, leading to a peak in the

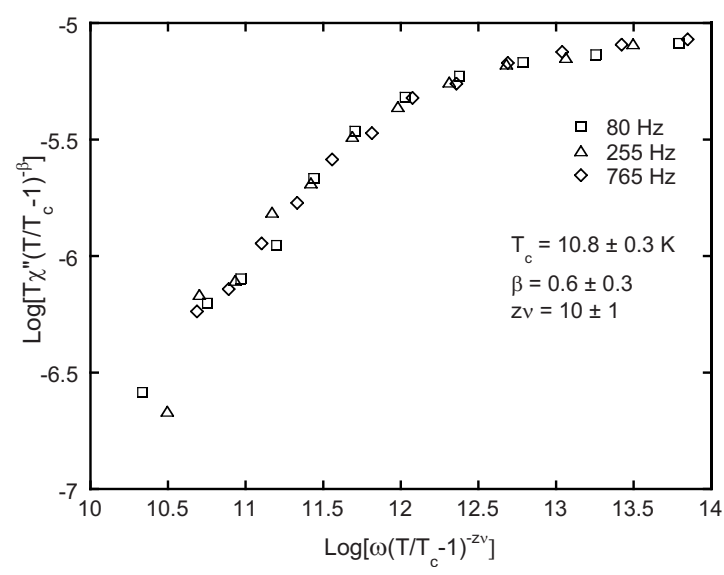

FIG. 3. Power-law dynamics scaling plot of the $\chi^{\prime \prime}(\omega, T)$ data for $\mathrm{Ga}_{1-x} \mathrm{Mn}_{x} \mathrm{~S}$, using the form given in Eq. (1) in the text. The best-fit values of the transition temperature $T_{\mathrm{c}}$ and the scaling exponents $z \nu$ and $\beta$ are shown.

susceptibility. Since smaller clusters are blocked at higher frequencies, the peak in the susceptibility occurs at higher temperatures for higher driving frequencies.

There is some scatter in our $\chi^{\prime \prime}(\omega, T)$ data because of the low level of the out-of-phase signal. This is especially apparent in the highest frequency $(2300 \mathrm{~Hz})$ data, which is omitted from the scaling analysis below. The lines in Fig. 2 are smoothing curves that were used to more precisely locate the peaks in the $\chi^{\prime \prime}(\omega, T)$ data, the positions of which are necessary for the scaling analysis that we now describe.

In the standard critical slowing down description of phase transitions, $\chi^{\prime \prime}(\omega, T)$ behaves according to the scaling $\operatorname{law}^{24,25}$

$$
T \chi^{\prime \prime}(\omega, T) \propto \varepsilon^{\beta} G\left(\omega \varepsilon^{-z \nu}\right),
$$

where $\beta$ is the critical exponent for the order parameter, $\varepsilon$ $=\left(T-T_{\mathrm{C}}\right) / T_{\mathrm{C}}$ quantifies the distance from the zero-frequency transition temperature $T_{\mathrm{C}}, z \nu$ is a product of critical exponents, and $G$ is a universal function of its argument. If the transition in $\mathrm{Ga}_{1-x} \mathrm{Mn}_{x} \mathrm{~S}$ is indeed that of a true spin glass, plotting the data according to Eq. (1) will cause data for all frequencies to collapse onto a single universal curve $(G)$ for the correct values of the critical exponents and $T_{\mathrm{C}}$. It should also be noted that on the basis of an analysis of the spin autocorrelation function, Mauger et al. ${ }^{26}$ have argued that Eq. (1) is only valid for temperatures $T>T_{p}$, where $T_{p}$ is the temperature at which the peak in $\chi^{\prime \prime}(\omega, T)$ occurs. We have taken this criterion into account in our analysis of all the $\chi^{\prime \prime}(\omega, T)$ data, which are shown in scaled form in Fig. 3. The data are well described by a single scaling curve. The best scaling was obtained for the following parameter values: $T_{\mathrm{c}}$ $=10.8 \pm 0.3 \mathrm{~K}, z \nu=10 \pm 1$, and $\beta=0.6 \pm 0.3$. The determination of the best collapse of the data was made by fitting the data to a fourth-order polynomial and calculating the chisquared statistic for each fit for a range of values of $T_{\mathrm{c}}, z \nu$, and $\beta$. The smallest value of chi-squared was obtained for the parameter values given above. Residuals were also checked to ensure that the best fits did not exhibit any pathological behavior. The uncertainties in the scaling exponents were obtained by ascertaining the extremal values of the pa- 


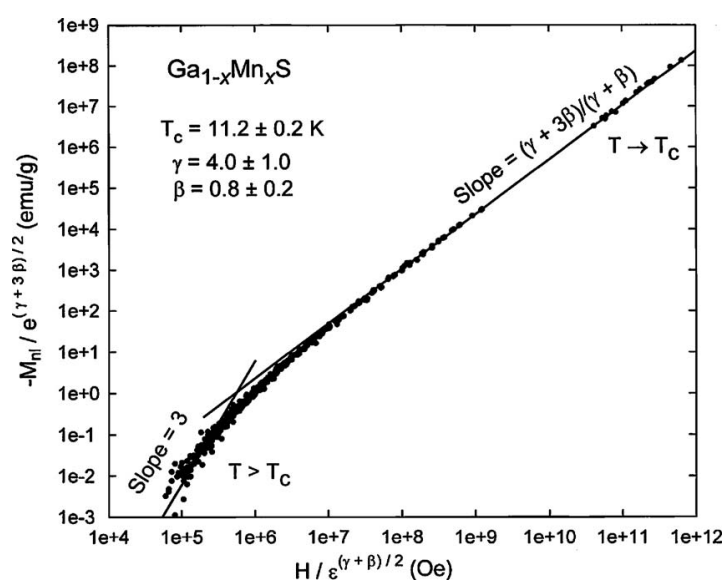

FIG. 4. The nonlinear magnetization data for $\mathrm{Ga}_{1-x} \mathrm{Mn}_{x} \mathrm{~S}$ analyzed according to a universal scaling model for a spin-glass transition [Eq. (2) in the text]. Magnetization versus temperature data taken in 100, 130, 160, 200, $250,300,400,500,750$, and 1000 Oe fields are shown. The asymptotic limits for the slope, shown by the solid lines, are in agreement with the theory. Note that the scaled nonlinear magnetization data for all ten set fields collapse onto the same universal scaling function over many orders of magnitude along both axes.

rameters for which reasonably good scaling was still achieved.

To unambiguously determine if the transition at $11.2 \mathrm{~K}$ in $\mathrm{Ga}_{1-x} \mathrm{Mn}_{x} \mathrm{~S}$ is due to a true spin-glass transition, ${ }^{22,27-29}$ we made nonlinear magnetization $M_{\mathrm{nl}}$ measurements on the $\mathrm{Ga}_{1-x} \mathrm{Mn}_{x} \mathrm{~S}$ sample close to the transition in order to examine the static scaling properties in the critical region. In the immediate vicinity above $T_{\mathrm{c}}$, the nonlinear magnetization follows the universal scaling expression

$$
M_{\mathrm{nl}}(\varepsilon, H)=\varepsilon^{(\gamma+3 \beta) / 2} F\left(H / \varepsilon^{(\gamma+\beta) / 2}\right),
$$

where $M_{\mathrm{nl}}$ is the nonlinear magnetization, $\beta$ is the critical exponent for the order parameter, $\gamma$ is another critical exponent, and $\varepsilon=\left(T-T_{\mathrm{c}}\right) / T_{\mathrm{c}}$ is the reduced temperature. ${ }^{26,30}$

To examine the behavior of $\mathrm{Ga}_{1-x} \mathrm{Mn}_{x} \mathrm{~S}$ near $T_{\mathrm{c}}$, measurements of the magnetization as a function of temperature were made in 10 fixed fields between 100 and 1000 Oe. In the region where $T>T_{\mathrm{c}},-\chi_{\mathrm{nl}}=-M_{\mathrm{nl}} / H$ increases, as one would expect for a spin-glass transition. In Fig. 4, the data for all ten fixed fields are plotted according to the universal scaling relation of Eq. (1) using values for the parameters $\gamma$, $\beta$, and $T_{\mathrm{c}}$ that produced the best scaling $(\gamma=4.0 \pm 1.0, \beta$ $=1.0 \pm 0.2$, and $T_{\mathrm{c}}=11.2 \pm 0.2 \mathrm{~K}$ ). The data collapse onto the single universal scaling curve that covers many orders of magnitude along both axes. For temperatures significantly greater than $T_{\mathrm{c}}$ (lower part of the curve), the slope approaches 3 as expected. The scaling plot shows increasing scatter at higher temperatures since $M_{\mathrm{nl}}$ approaches zero as the temperature increases above the critical region. As $T$ closely approaches $T_{\mathrm{c}}$ from above (upper part of the curve), the slope tends to the proper asymptotic value $(\gamma+3 \beta) /(\gamma$ $+\beta) .{ }^{26}$ These asymptotic limits are shown as solid lines in Fig. 4. Data were taken for additional fixed fields ranging from 2 to 75 Oe. At these lower fields, the nonlinear magnetization data follow the same scaling function, but are not plotted because of decreasing signal to noise for data taken in the lower fields.
Magnetization and ac susceptibility measurements on single crystalline $\mathrm{Ga}_{1-x} \mathrm{Mn}_{x} \mathrm{~S}$ ( $\left.x=0.09\right)$ have been measured near the spin-glass transition. No other III-VI DMS is currently known to exhibit a spin-glass transition for comparison with $\mathrm{Ga}_{1-x} \mathrm{Mn}_{x} \mathrm{~S}$. Both the magnetization and ac susceptibility measurements were analyzed according to the appropriate universal scaling function for spin-glass transitions. The nonlinear magnetization scaled with the critical exponent values $\gamma=4.0 \pm 1.0$ and $\beta=0.8 \pm 0.2$ for a spinglass transition temperature $T_{\mathrm{c}}=11.2 \pm 0.2 \mathrm{~K}$. The analysis of the ac susceptibility's out-of-phase component $\chi^{\prime \prime}(\omega, T)$ yielded the parameter values $T_{\mathrm{c}}=10.8 \pm 0.3 \mathrm{~K}, z \nu=10 \pm 1$, and $\beta=0.6 \pm 0.3$.

The values of the critical exponents are consistent with those obtained in many DMS and non-DMS insulating spin glasses with different lattice structures and exchange interactions. ${ }^{22}$ Our results therefore lead us to conclude that III-V DMS materials such as $\mathrm{Ga}_{1-x} \mathrm{Mn}_{x} \mathrm{~S}$ belong in the same three-dimensional short-range Heisenberg universality class as other DMS and non-DMS insulating spin glasses. We also note that similar exponents have been found in the 3D Ising system $\mathrm{Fe}_{0.5} \mathrm{Mn}_{0.5} \mathrm{TiO}_{3}{ }^{31}$ This may indicate Ising-like character in supposed Heisenberg systems, possibly due to the presence of anisotropy.

This research was supported by ACS-PRF Grant No. 40209-B5M, a Purdue Univ. Academic Reinvestment Program, and by the National Science Foundation (NSF) Grant Nos. DMR-07-06593 and DMR-04-05082.

${ }^{1}$ T. M. Pekarek et al., J. Appl. Phys. 83, 6557 (1998).

${ }^{2}$ N. C. Fernelius, Prog. Cryst. Growth Charact. Mater. 28, 275 (1994).

${ }^{3}$ D. R. Suhre et al., Opt. Lett. 22, 775 (1997).

${ }^{4}$ M. O. D. Camara et al., Phys. Rev. B 65, 205308 (2002).

${ }^{5}$ I. H. Choi and P. Y. Yu, Phys. Rev. B 68, 165339 (2003).

${ }^{6}$ J. Z. Wan, J. L. Brebner, and R. Leonelli, Phys. Rev. B 53, 15413 (1996).

${ }^{7}$ F. J. Manjón, A. Segura, and V. Muñoz, J. Appl. Phys. 81, 6651 (1997).

${ }^{8}$ A. B. M. O. Islam et al., J. Appl. Phys. 85, 4003 (1999).

${ }^{9}$ N. Okamotoet al., Appl. Phys. Lett. 73, 794 (1998).

${ }^{10}$ M. Budiman et al., Jpn. J. Appl. Phys., Part 1 37, 5497 (1998).

${ }^{11}$ M. A. Alzhdanovet al., Phys. Solid State 41, 20 (1999).

${ }^{12}$ N. Okamoto et al., Jpn. J. Appl. Phys., Part 1 37, 3248 (1998).

${ }^{13}$ T. M. Pekarek et al., J. Appl. Phys. 87, 6448 (2000).

${ }^{14}$ C. Fuller et al., Phys. Rev. B 65, 195211 (2002).

${ }^{15}$ J. L. Tracy et al., J. Appl. Phys. 99, 08D507 (2006).

${ }^{16}$ J. L. Tracy et al., Phys. Rev. B 72, 165201 (2005).

${ }^{17}$ T. M. Pekarek et al., J. Appl. Phys. 99, 08D511 (2006).

${ }^{18}$ D. Meda et al., J. Appl. Phys. 105, 07C521 (2009).

${ }^{19}$ T. M. Pekarek et al., J. Appl. Phys. 89, 7030 (2001).

${ }^{20}$ Semiconductors and Semimetals, edited by J. K. Furdyna and J. Kossut (Academic, Boston, 1988), Vol. 25.

${ }^{21}$ Spin Glasses An Experimental Introduction, edited by J. A. Mydosh (Taylor and Francis, Washington, DC, 1993).

${ }^{22}$ P. M. Shand et al., Phys. Rev. B 58, 12876 (1998).

${ }^{23}$ T. M. Pekarek et al., J. Appl. Phys. 101, 09D511 (2007).

${ }^{24}$ Y. Zhou et al., Phys. Rev. B 40, 8111 (1989).

${ }^{25}$ M. Continentino and A. P. Malozemoff, Phys. Rev. B 34, 471 (1986).

${ }^{26}$ A. Mauger, J. Ferre, and P. Beauvillain, Phys. Rev. B 40, 862 (1989).

${ }^{27}$ J. A. Mydosh, Spin Glasses: An Experimental Introduction (Taylor and Francis, London, 1993).

${ }^{28}$ Spin Glasses, in Cambridge Studies in Magnetism:1, edited by D. Edwards and D. Melville (Cambridge University Press, Cambridge, 1991).

${ }^{29}$ A. Mauger et al., Phys. Rev. B 41, 4587 (1990) (and references therein).

${ }^{30}$ A. P. Ramirez et al., Phys. Rev. Lett. 64, 2070 (1990).

${ }^{31}$ K. Gunnarsson et al., Phys. Rev. Lett. 61, 754 (1988). 